Rev. Int. Contam. Ambie. 36 (3) 517-528, 2020

https://doi.org/10.20937/RICA.53520

\title{
ESTUDIO DE LOS EFECTOS DE DIFERENTES OPCIONES DE FERTILIZACIÓN Y ROTACIONES SOBRE EL CULTIVO DE CAMELINA EN UNA REGIÓN SEMIÁRIDA DE ESPAÑA
}

Study of the effects of different fertilization options and rotations on crop camelina in a semiarid region of Spain

\author{
José Valero MARTÍN SÁNCHEZ1*, María Isabel GONZÁLEZ GULLÓN", \\ Gonzalo HERNANDO ÁLVAREZ², Javier PRIETO RUIZ², \\ Aníbal CAPUANO ${ }^{2}$ y María del Mar DELGADO ARROYO ${ }^{1}$
}

${ }^{1}$ Departamento de Medio Ambiente, Instituto Nacional de Investigación y Tecnología Agraria y Alimentaria, km 7.5 carretera La Coruña, 28040 Madrid, España

${ }^{2}$ Camelina Company España, Camino de la Carrera 11, 28140 Fuente el Saz del Jarama, Madrid, España

*Autor para correspondencia: vmartin@inia.es

(Recibido enero 2019; aceptado octubre 2019)

Palabras clave: fertilizante orgánico, lodos de depuradora, compost, germinación, rendimientos

\section{RESUMEN}

Se realizó un estudio durante tres campañas sobre el cultivo de camelina (Camelina sativa L. Crantz) para evaluar su respuesta frente a varios tipos de fertilización: lodo deshidratado, lodo compostado y fertilización mineral. También se evaluó su rotación con el cultivo de cebada (Hordeum vulgare L.). La siembra se realizó en otoño y se la cosecha en primavera-verano. La germinación del cultivo de camelina fue mayor con la fertilización de lodo deshidratado frente a la fertilización mineral en las tres campañas. La germinación del cultivo de cebada fue menor con la fertilización mineral en la primera campaña y con el testigo en la tercera campaña frente al resto de tratamientos. El desarrollo del ciclo de ambos cultivos (camelina y cebada) estuvo muy influenciado por las condiciones meteorológicas. Se obtuvieron muy bajos rendimientos en la tercera campaña debido a baja pluviometría en primavera y altas temperaturas al final del ciclo de cultivo. La camelina presentó mayor producción con la fertilización de lodo deshidratado frente al testigo y lodo compostado tras la primera campaña. La cebada también presentó las mayores producciones con lodo deshidratado frente al mineral y sin diferencias significativas frente al lodo compostado. Tras las tres campañas agrícolas de cultivo, los tratamientos fertilizantes aumentaron las cantidades de nitrógeno, fosforo y potasio en el suelo frente al testigo y la fertilización orgánica mejoró parámetros del suelo como la materia orgánica. Los mayores rendimientos económicos fueron para el cultivo sin fertilizar y el lodo deshidratado frente al resto de tratamientos.

Key words: organic fertilizer, sewage sludge, compost, germination, yields

\begin{abstract}
A study was carried out for three growing seasons on camelina (Camelina sativa $\mathrm{L}$. Crantz) to evaluate its response to different fertilizers: dehydrated sewage sludge, composted sewage sludge and mineral fertilization, as well as its rotation with barley
\end{abstract}


(Hordeum vulgare L.) and fallow. The camelina crop was sown in autumn and harvested in spring-summer; its germination was greater with dehydrated sewage sludge fertilization than with mineral fertilization in all seasons. The germination of the barley crop was lower with the mineral fertilization in the first growing season and with the control in the third season compared to the rest of the treatments. The development of camelina and barley was very much influenced by meteorological conditions. The last growing season had the lowest productivity due to limited rainfall and high temperature at the end of this growing season. The camelina presented the larger yields with fertilization of dehydrated sewage sludge than with control and composted sewage sludge after the first season. Barley also presented the largest productions with dehydrated sewage sludge compared to the mineral and without significant differences compared to the composted sewage sludge. After the three growing seasons, fertilizer treatments increased the amounts of nitrogen, phosphorus and potassium in the soil compared to control and organic fertilization improved soil parameters such as organic matter. The highest economic yields were for the unfertilized crop and the dehydrated sewage sludge compared to the rest of the treatments.

\section{INTRODUCCIÓN}

La camelina (Camelina sativa L. Crantz), conocida como falso lino, es una especie oleaginosa perteneciente a la familia Brassicaceae que tiene un gran potencial como materia prima de bajo coste para la producción de biodiesel en muchas regiones del mundo. Los principales productos que se obtienen a partir de este cultivo son: aceite con alto contenido en ácidos grasos insaturados (15\% linoleico y $38 \%$ linolénico; Zubr 2007), cuya materia prima tiene como aprovechamiento principal su uso como combustible de aviación; y harina con alto contenido proteico, la cual que se usa en la producción de piensos para alimentación animal.

La camelina es un cultivo oleaginoso que no compite con el sector alimentario, ya que tiene requerimientos nutricionales bajos en cuanto a macronutrientes (Wojtkowiak et al. 2009); además, contribuye a disminuir los problemas de emisiones de gases de efecto invernadero porque se cultiva en terrenos marginales o en rotaciones de cereales sustituyendo al barbecho. Tiene un considerable potencial agronómico y ecológico para reemplazar a este último, por lo cual se aprovecha ya en sistemas agrícolas de Estados Unidos (Keshavarz-Afshar et al. 2016) con ese fin. Este cultivo también se caracteriza por su tolerancia a las heladas y a la sequía, y se puede mecanizar íntegramente con maquinaria comercial empleada en el cultivo de cereales de invierno.

Por otra parte, con motivo de cumplir con lo establecido en la Directiva 91/271/CEE (CCE 1991) sobre tratamiento de aguas residuales urbanas, a través del RD-Ley 11/1995 (BOE 1995), los municipios españoles tienen la obligación de adoptar las medidas necesarias para garantizar que antes de su vertido dichas aguas sean tratadas correctamente en instalaciones adecuadas, con el fin último de garantizar la protección del medio ambiente. Este proceso de depuración al que han de someterse las aguas residuales urbanas lleva aparejada la generación de lodos de depuradora que, según la citada directiva, han de gestionarse y tratarse de forma adecuada, siendo las limitaciones ambientales cada vez mayores y los métodos de tratamiento cada vez más restrictivos como resultado de la legislación de aplicación establecida por la Orden AAA/1072/2013 (BOE 2013).

La aplicación de la Directiva 91 ha promovido un incremento de la producción de lodos en la Unión Europea (UE), de 6.5 millones de t de materia seca en 1992 a 10.9 en 2005, y se estima que supere los 13 millones de t de materia seca en 2020 (Leonard 2011, Kelessidis y Stasinakis 2012).

En la actualidad, las estaciones de tratamiento de aguas residuales urbanas (EDAR) generan miles de toneladas de lodos. Los tres contribuyentes más importantes de lodos sólidos secos son la Unión Europea con 11.7 millones de t (2010), China con 9.18 millones de t (2009) y Estados Unidos con más de ocho millones de t (2011) (Arlabosse et al. 2012, Bennamoun et al. 2013). La eliminación de lodos representa todavía de 25 a $65 \%$ de los costes totales de gestión en una EDAR (Pérez-Elvira et al. 2006).

En las estaciones depuradoras se conocen en conjunto como lodos frescos los lodos no tratados. Una vez que se les somete a tratamientos específicos de la línea de fangos (espesamiento, estabilización, deshidratación) se les denomina fangos espesados, estabilizados o digeridos, y lodos deshidratados (LD), respectivamente. Los más usados son los LD y los lodos compostados (LC) (Fytili y Zabaniotou 2008). 
Los LD son aquellos que se someten a un proceso de pérdida de agua por procedimientos físicos, químicos o térmicos, antes de su utilización. Su contenido de humedad no debe superar el $80 \%$.

Los LC se someten a un proceso de transformación biológica aerobia, con la finalidad de obtener un producto estable y no fitotóxico. El compostaje de lodos es una alternativa necesaria para cubrir los periodos en los que no es posible realizar la aplicación directa de los lodos; además, es una alternativa económicamente viable y ambientalmente segura para la estabilización de la materia orgánica de los lodos de EDAR, especialmente, para su utilización posterior en agricultura, y una forma de sanearlos de microorganismos patógenos u otras sustancias fitotóxicas.

Desde un punto de vista práctico, el lodo es una valiosa fuente de carbono orgánico, nitrógeno y fósforo, así como de algunos compuestos inorgánicos como silicatos, aluminatos, etc., que puede ser reciclado y utilizado para fines agrícolas (Dentel 2004, Fytili y Zabaniotou 2008). El principal riesgo asociado a la aplicación de lodos en la agricultura es el potencial de acumulación a largo plazo de ciertos elementos tóxicos (Singh y Agrawal 2008, Uggetti et al. 2012).

El objetivo del presente trabajo fue determinar los efectos de la fertilización orgánica en forma de LD y LC frente a la fertilización mineral, para su uso en el cultivo de camelina cv V11 y estudiar su comportamiento a lo largo del ciclo del cultivo, así como en un cultivo de cebada (Hordeum vulgare L.) cv Vinagrosa en rotación con la camelina.

\section{MATERIALES Y MÉTODOS}

La investigación se llevó a cabo en el Laboratorio de Depuración y Reutilización Agraria de Residuos Ganaderos y Urbanos del Instituto Nacional de Investigación y Tecnología Agraria y Alimentaria (INIA), en una parcela de campo sita en la finca experimental La Canaleja en Alcalá de Henares, provincia de Madrid, cuyas coordenadas geográficas son $40^{\circ} 30^{\prime}$ $\mathrm{N}$ y $3^{\circ} 18^{\prime} \mathrm{O}$, con una altitud de $600 \mathrm{~m}$. El suelo se clasifica como Haploxeralf según clasificación taxonómica de suelos del United States Department of Agriculture (Soil Survey Staff 2010).

El ensayo se realizó en una parcela de campo experimental de 1.4 ha, la cual se dividió en dos bloques para realizar la rotación entre los dos cultivos a ensayar, camelina y cebada. Dentro de cada bloque se situaron 12 parcelas con los diferentes tratamientos, situadas al azar, con tres repeticiones por tratamiento. Las dimensiones de cada parcela fueron de $20 \times 10 \mathrm{~m}$. Los tratamientos y dosis ensayados fueron: testigo (T), fertilización mineral (M) $(300 \mathrm{~kg} /$ ha por campaña), LD (40 t/ha para las tres campañas) y LC ( $8 \mathrm{t} / \mathrm{ha}$ por campaña), para obtener una dosis equivalente de 180 unidades fertilizantes de nitrógeno (UFN) para el total de las tres campañas. La aplicación se realizó un mes antes de la siembra en el caso de los lodos, los cuales se incorporaron al suelo mediante una labor, mientras que la fertilización mineral de fondo con una dosis de $100 \mathrm{~kg} / \mathrm{ha}(8-24-8)$ se aplicó 10 días antes de la siembra, incorporándose con la labor de preparación del terreno para la siembra. En las tres campañas, al inicio de la elongación del tallo en el cultivo de camelina en la primera semana de marzo, se procedió al abonado de cobertera en el tratamiento de fertilización mineral con una dosis de $200 \mathrm{~kg} / \mathrm{ha}$ de nitrosulfato amónico (26-0-0) con $37 \%$ de tiosulfato; se alcanzó una dosis de 60 UFN por campaña para una producción de cebada esperada de $2500 \mathrm{~kg} / \mathrm{ha}$.

El estudio se realizó durante tres campañas en 2014-2015; 2015-2016 y 2016-2017, en las que se produjeron las rotaciones de cultivo (camelinacebada) en dos bloques cada uno, con todos los tratamientos fertilizantes y tres repeticiones por tratamiento. Las aplicaciones de fertilización mineral y LC se efectuaron sobre la misma parcela en cada una de las tres campañas, en tanto que para el LD se realizó una única aplicación en la primera campaña.

Antes de la aplicación de los residuos se tomó una muestra de suelo de cada parcela, a $0-30 \mathrm{~cm}$ y a 30-60 cm de profundidad, y se realizó su caracterización para ajustar las dosis de fertilización según las necesidades de los cultivos a ensayar.

Después de cada campaña se tomaron muestras de suelo para conocer su evolución. Los parámetros físicos y químicos obtenidos se muestran en el cuadro I para el suelo inicial y en el cuadro II para el suelo final, tras la tercera campaña.

Durante las campañas se registraron los siguientes datos climatológicos: humedad relativa, temperatura, precipitación, radiación fotosintéticamente activa (PAR), evapotranspiración de referencia (ETo), velocidad y dirección del viento. Las mediciones se obtuvieron de una estación situada en la propia finca experimental, situada a $200 \mathrm{~m}$ de la parcela de ensayo.

La fecha de siembra óptima para estos cultivos en la zona en que se desarrolló el ensayo es el otoño. En las dos primeras campañas del estudio la siembra se realizó la primera semana de noviembre y en la tercera campaña en la última semana de octubre, tanto en el caso de la camelina como en el de la cebada. 
CUADRO I. CARACTERÍSTICAS DE LOS LODOS Y SUELOS INICIALES PARA CADA TRATAMIENTO

\begin{tabular}{|c|c|c|c|c|c|c|}
\hline \multirow{2}{*}{ Parámetros } & \multicolumn{2}{|c|}{ Lodos } & \multicolumn{4}{|c|}{ Suelos } \\
\hline & LD & $\mathrm{LC}$ & $\mathrm{T}$ & M & $\mathrm{LC}$ & LD \\
\hline 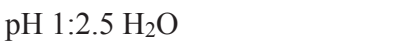 & 7.61 & 8.11 & 8.39 & 8.36 & 8.26 & 8.11 \\
\hline $\mathrm{CE} 1: 5 \mathrm{H}_{2} \mathrm{O} \mathrm{dS} / \mathrm{m}$ & 3.97 & 4.74 & 0.127 & 0.139 & 0.143 & 0.181 \\
\hline C orgánico oxidable $(\%)$ & 16.87 & 18.45 & 0.93 & 1.05 & 1.12 & 1.08 \\
\hline Materia orgánica oxidable (\%) & 27.86 & 33.01 & 1.61 & 1.81 & 1.93 & 1.86 \\
\hline Nitrógeno Kjeldahl (\%) & 5.91 & 2.05 & 0.101 & 0.112 & 0.128 & 0.124 \\
\hline $\mathrm{N}-\mathrm{NH}_{4}^{+}(\mathrm{mg} / \mathrm{kg})$ & 16350.4 & 4372.8 & 2.52 & 3.41 & 2.55 & 3.11 \\
\hline $\mathrm{N}-\mathrm{NO}_{3}{ }^{-}(\mathrm{mg} / \mathrm{kg})$ & 345.33 & 818.922 & 17.321 & 25.941 & 35.42 & 55.911 \\
\hline $\mathrm{P}_{2} \mathrm{O}_{5}$ total $(\%)$ & 3.56 & 4.4 & 0.176 & 0.15 & 0.31 & 0.23 \\
\hline $\mathrm{K}_{2} \mathrm{O}$ total $(\%)$ & 0.72 & 0.73 & 0.52 & 0.59 & 0.5 & 0.52 \\
\hline $\mathrm{CaO}$ total $(\%)$ & 6.23 & 7.54 & 4.72 & 5.96 & 5.15 & 6.25 \\
\hline $\mathrm{MgO}$ total $(\%)$ & 1.32 & 1.58 & 0.94 & 1.21 & 1.03 & 1.11 \\
\hline $\mathrm{Na}_{2} \mathrm{O}$ total $(\%)$ & 0.25 & 0.21 & 0.057 & 0.085 & 0.062 & 0.07 \\
\hline $\mathrm{Zn}(\mathrm{mg} / \mathrm{kg})$ & 540 & 511 & 57.2 & 67.5 & 78.65 & 82.61 \\
\hline $\mathrm{Cu}(\mathrm{mg} / \mathrm{kg})$ & 337 & 168 & 20.9 & 28.8 & 33.4 & 38.52 \\
\hline $\mathrm{Cr}(\mathrm{mg} / \mathrm{kg})$ & 24 & 54 & 9.1 & 8.6 & 15.4 & 10.22 \\
\hline $\mathrm{Ni}(\mathrm{mg} / \mathrm{kg})$ & 19 & 25.2 & 10.6 & 11.12 & 11.19 & 10.91 \\
\hline $\mathrm{Pb}(\mathrm{mg} / \mathrm{kg})$ & 35 & 36.4 & 24.36 & 29.14 & 34.9 & 37.44 \\
\hline $\mathrm{Cd}(\mathrm{mg} / \mathrm{kg})$ & 2 & 0.75 & 0.15 & 0.75 & 0.8 & 1.04 \\
\hline
\end{tabular}

T: testigo, M: fertilización mineral, LC: lodo compostado, LD: lodo deshidratado, CE: conductividad eléctrica

CUADRO II. CARACTERÍSTICAS DE LOS SUELOS FINALES PARA CADA TRATAMIENTO

\begin{tabular}{|c|c|c|c|c|}
\hline \multirow{2}{*}{ Parámetros } & \multicolumn{4}{|c|}{ Suelos } \\
\hline & $\mathrm{T}$ & M & $\mathrm{LC}$ & LD \\
\hline $\mathrm{pH} 1: 2.5 \mathrm{H}_{2} \mathrm{O}$ & 8.34 & 8.22 & 8.2 & 8.27 \\
\hline $\mathrm{CE} 1: 5 \mathrm{H}_{2} \mathrm{O}(\mathrm{dS} / \mathrm{m})$ & 0.102 & 0.179 & 0.126 & 0.145 \\
\hline C orgánico oxidable (\%) & 1.03 & 1.09 & 1.17 & 1.21 \\
\hline Materia orgánica oxidable (\%) & 1.76 & 1.87 & 2.03 & 2.08 \\
\hline Nitrógeno Kjeldahl (\%) & 0.081 & 0.137 & 0.108 & 0.133 \\
\hline $\mathrm{N}-\mathrm{NH}_{4}^{+}(\mathrm{mg} / \mathrm{kg})$ & 4.16 & 6.68 & 3.89 & 6.21 \\
\hline $\mathrm{N}^{-\mathrm{NO}_{3}}{ }^{-}(\mathrm{mg} / \mathrm{kg})$ & 13.93 & 66.97 & 18.52 & 21.44 \\
\hline $\mathrm{P}_{2} \mathrm{O}_{5}$ total $(\%)$ & 0.244 & 0.285 & 0.264 & 0.28 \\
\hline $\mathrm{K}_{2} \mathrm{O}$ total $(\%)$ & 0.73 & 0.80 & 0.71 & 0.72 \\
\hline $\mathrm{CaO}$ total $(\%)$ & 4.11 & 3.23 & 4.81 & 3.31 \\
\hline $\mathrm{MgO}$ total $(\%)$ & 1.14 & 1.09 & 1.22 & 1.06 \\
\hline $\mathrm{Na}_{2} \mathrm{O}$ total $(\%)$ & 0.126 & 0.111 & 0.136 & 0.115 \\
\hline $\mathrm{Zn}(\mathrm{mg} / \mathrm{kg})$ & 75.24 & 97.04 & 97.71 & 110.01 \\
\hline $\mathrm{Cu}(\mathrm{mg} / \mathrm{kg})$ & 31.49 & 28.18 & 36.01 & 45.52 \\
\hline $\mathrm{Cr}(\mathrm{mg} / \mathrm{kg})$ & 13.76 & 12.06 & 12.81 & 13.26 \\
\hline $\mathrm{Ni}(\mathrm{mg} / \mathrm{kg})$ & 11.29 & 11.48 & 11.31 & 11.52 \\
\hline $\mathrm{Pb}(\mathrm{mg} / \mathrm{kg})$ & 23.89 & 31.67 & 24.73 & 35.39 \\
\hline $\mathrm{Cd}(\mathrm{mg} / \mathrm{kg})$ & 0.39 & 0.58 & 0.79 & 0.79 \\
\hline
\end{tabular}

T: testigo, M: fertilización mineral, LC: lodo compostado, LD: lodo deshidratado, CE: conductividad eléctrica

La cebada utilizada fue Hordeum vulgare L. cv Vinagrosa con una dosis de siembra de $200 \mathrm{~kg} / \mathrm{ha}$. Para la camelina se usó Camelina sativa L. Crantz cv V11, con una dosis de siembra de $8 \mathrm{~kg} / \mathrm{ha}$.
Para efectuar el seguimiento de los cultivos se instalaron aros con un diámetro de $60 \mathrm{~cm}$ en el centro de cada parcela y se tomaron sus coordenadas mediante el sistema de posicionamiento global 
(GPS). Se siguió un protocolo en el que se registraron las fechas de germinación, roseta, inicio de elongación de tallo, floración, maduración-llenado de grano y cosecha. Igualmente se controló el número de plantas dentro de cada aro, registrando los datos quincenalmente.

El diseño estadístico del ensayo fue en bloques al azar con cuatro tratamientos fertilizantes para cada cultivo (camelina y cebada) durante tres campañas. Para el estudio estadístico se realizó una comprobación de la normalidad de los datos; posteriormente se realizaron un análisis de varianza multifactorial y un estudio para los factores tratamiento, campaña y sus interacciones, con el fin de evaluar la influencia tratamiento por campaña. Los datos se analizaron con el programa Statgraphics Centurión v. XV II. Para la separación de medias se utilizó, en todos los casos, la prueba de rango múltiple de Duncan con un nivel de significación $\alpha=0.05$. Para el estudio de los efectos en el suelo se realizó un análisis de varianza comparando los parámetros físicos y químicos estudiados de los suelos tras cada cultivo (camelina y cebada), y otro comparando dichos parámetros en el suelo inicial y el final tras las tres campañas.

\section{RESULTADOS Y DISCUSIÓN}

La germinación del cultivo de la camelina se produjo 10 días después de la siembra en la primera y tercera campaña. En el caso de la cebada, la germinación se demoró una semana más (17 días) en estas campañas. Para la segunda campaña, en ambos cultivos se produjo la germinación en dos fechas, una a los 25 días de la siembra y otra a los 68 días de la siembra.

Dentro del seguimiento quincenal se efectuó un primer conteo del número de plantas al mes de la germinación y un segundo conteo a los dos meses y medio de la germinación. En general, en el primer conteo no se apreciaron diferencias significativas en el número de plantas $/ \mathrm{m}^{2}$ entre tratamientos para ambos cultivos, excepto en la segunda campaña, debido a un retraso en la germinación y también a que dicha germinación se produjo en dos fechas. En este control no se encontraron diferencias significativas en el número de plantas $/ \mathrm{m}^{2}$ para el cultivo de camelina, pero sí para el cultivo de cebada, en el que el tratamiento residual de LD fue inferior a la M y el T. Sin embargo, en la segunda germinación de la camelina sí se encontraron diferencias significativas, el residual del LD fue mayor en comparación con el tratamiento $\mathrm{M}$ y no hubo diferencias en el cultivo de cebada.
En el cuadro III se puede apreciar que para el caso del cultivo de camelina la fertilización con LD en las tres campañas produjo el mayor número de plantas frente al resto de los tratamientos, con diferencias significativas respecto a la fertilización mineral. A pesar de que sólo se fertilizó con LD en la primera campaña, en la última campaña el efecto residual siguió manteniendo las diferencias frente al resto de tratamientos. Esta disminución del número de plantas al aumentar la fertilización mineral también fue observada por autores como Jiang y Caldwell (2016) y Bobrecka-Jamro (2017). Con relación al cultivo de cebada, sólo se produjeron diferencias significativas entre el LD y la fertilización mineral en la primera campaña y respecto al $\mathrm{T}$ en la tercera campaña.

CUADRO III. NÚMERO DE PLANTAS POR METRO CUADRADO SEGÚN TRATAMIENTOS Y CULTIVOS

\begin{tabular}{|c|c|c|c|c|c|}
\hline \multirow{2}{*}{ Campañas } & \multirow{2}{*}{ Cultivos } & \multicolumn{4}{|c|}{ Tratamientos } \\
\hline & & $\mathrm{T}$ & M & $\mathrm{LC}$ & LD \\
\hline 1 & Camelina & $209 \mathrm{ab}$ & $113 \mathrm{~b}$ & $148 \mathrm{ab}$ & $246 \mathrm{a}$ \\
\hline 1 & Cebada & $171 \mathrm{a}$ & $117 \mathrm{~b}$ & $183 \mathrm{a}$ & $161 \mathrm{a}$ \\
\hline 2 & Camelina & $226 \mathrm{ab}$ & $185 \mathrm{~b}$ & $257 \mathrm{ab}$ & $278 \mathrm{a}$ \\
\hline 2 & Cebada & $175 \mathrm{a}$ & $166 \mathrm{a}$ & $182 \mathrm{a}$ & $144 \mathrm{a}$ \\
\hline 3 & Camelina & $103 \mathrm{~b}$ & $103 \mathrm{~b}$ & $97 \mathrm{~b}$ & $140 \mathrm{a}$ \\
\hline 3 & Cebada & $134 \mathrm{~b}$ & $228 \mathrm{a}$ & $261 \mathrm{a}$ & $265 \mathrm{a}$ \\
\hline
\end{tabular}

T: testigo, M: fertilización mineral, LC: lodo compostado, LD: lodo deshidratado

Nota: Los valores con letras iguales en cada fila no presentan diferencias significativas según la prueba de Duncan $(\alpha=0.05)$

Respecto del cultivo de camelina, si se comparan las campañas sin tener en cuenta los tratamientos, el mayor número de plantas $/ \mathrm{m}^{2}$ se obtuvo en la segunda campaña con 237, seguida de la primera con 179 y por último la tercera con 111, encontrándose diferencias significativas entre ellas. Estas densidades se encuentran dentro de los rangos normales (111-280 plantas $/ \mathrm{m}^{2}$ ) de acuerdo con autores como Gesch (2014). Con relación a la cebada se produjeron diferencias significativas entre la tercera campaña con 220 y la segunda y primera campañas, con 166 y 158 plantas $/ \mathrm{m}^{2}$, respectivamente.

La elongación del tallo se observó en el cuarto mes después de la siembra, por lo que en la primera semana de marzo se procedió, en las tres campañas, al abonado de cobertera en las parcelas con el tratamiento de fertilización mineral. La floración comenzó unos 20 días después de dicha elongación en las 
dos primeras campañas, y después de 10 días en la tercera; también hubo diferencias entre las tres campañas estudiadas respecto a la duración y el tiempo de llenado del grano. Estas diferencias se debieron a variaciones climatológicas entre campañas, como se verá a continuación.

El ciclo de cultivo de mayor duración ocurrió en la segunda campaña (220 días) debido a la germinación en dos etapas. Las diferencias en el desarrollo de las plantas debidas a su germinación produjeron una floración más larga (20 días), y lo mismo ocurrió con el llenado del grano (28 días). El ciclo de cultivo más corto se observó en la tercera campaña (190 días), ya que en menos de un mes ocurrieron tanto la floración del cultivo (siete días) como el llenado del grano (10 días). El cultivo de cebada tuvo un comportamiento similar en cuanto a la duración de los ciclos de cultivo, con 215, 240 y 205 días para la primera, segunda y tercera campañas, respectivamente. Estos ciclos más cortos tienen un efecto sobre la producción. Según Pervez-Zamurrad et al. (2010), la reducción del periodo de crecimiento en ciertas fases del ciclo (como la formación de la cubierta vegetal) reduce el tiempo de vernalización y de formación de los granos.

La cosecha de camelina se llevó a cabo a finales de mayo en la primera campaña, a mediados de junio en la segunda y a inicios de mayo en la tercera campaña. Respecto de la cebada, ocurrió 15 días después de la cosecha de camelina en las tres campañas.
Para comprobar que las variaciones climatológicas pudieran justificar estos cambios en la duración de los ciclos de cultivo, así como las diferencias en la producción que se abordarán más adelante, se realizó un análisis de los datos meteorológicos en las tres campañas. El resumen de varios parámetros de los datos meteorológicos se muestra en la figura 1.

El aumento de las temperaturas en la tercera campaña frente a la segunda y la falta de precipitación en la tercera campaña frente a la primera y la segunda en las fases de elongación y llenado, junto con otros parámetros como ETo alta y baja humedad relativa en ese periodo, fueron los causantes de las diferencias encontradas, tanto en la duración como en las producciones de la tercera campaña. El estudio de Warrick (1988) para EUA, Reino Unido y Europa occidental sobre el aumento de la temperatura en la productividad del trigo indica que su impacto es catastrófico en términos de pérdidas de rendimiento porque una mayor temperatura acelera el proceso de evapotranspiración, lo que genera estrés por humedad.

Las figuras 2 y 3 muestran las producciones obtenidas de cada cultivo para los diferentes tratamientos y campañas, así como las diferencias significativas encontradas, según la prueba de Duncan $(\alpha=0.05)$, entre tratamientos en cada campaña.

Con el fin de analizar conjuntamente el efecto ejercido sobre el rendimiento de la cosecha, se ha calculado la producción total, tanto de grano como

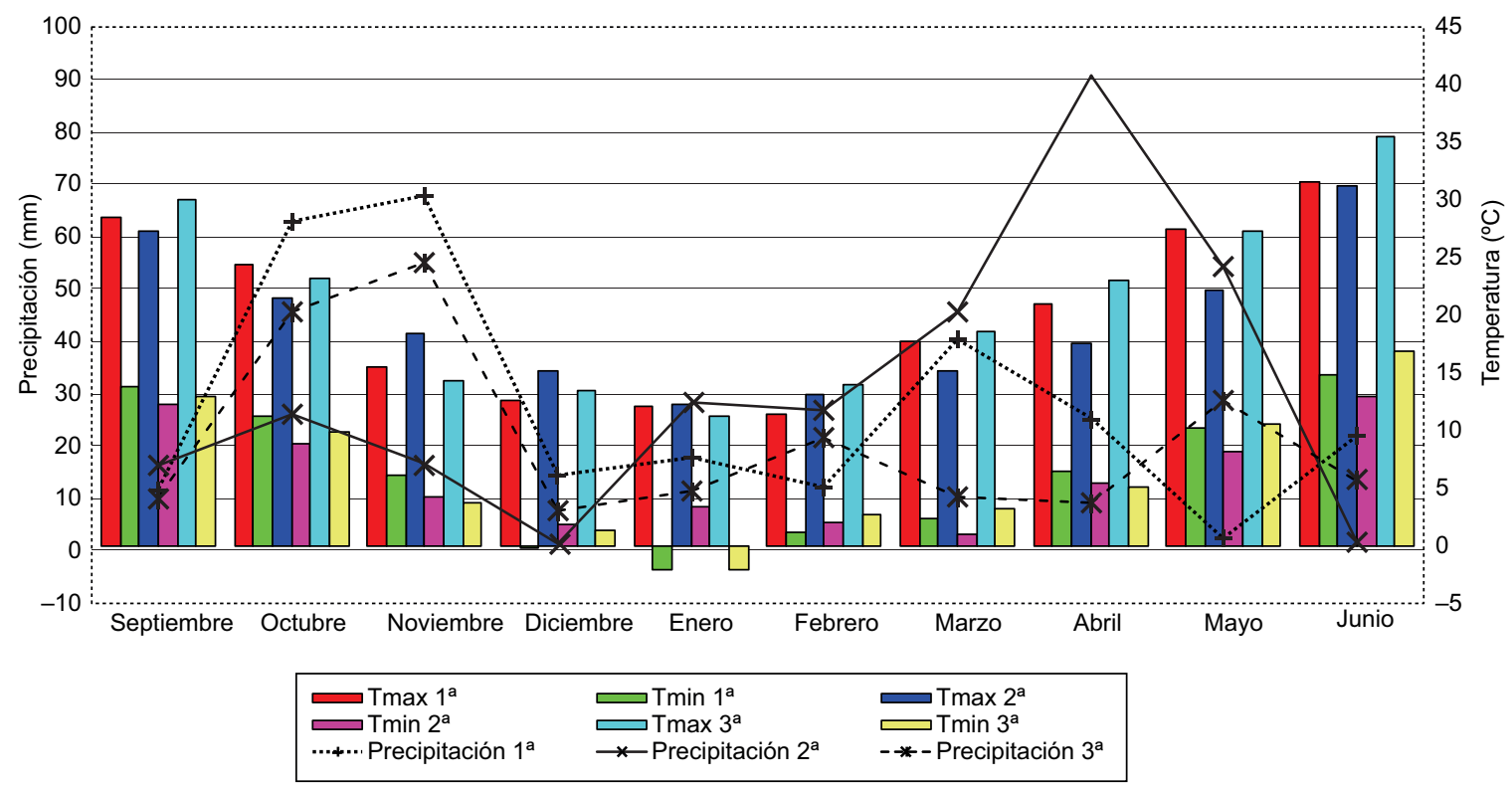

Fig. 1. Datos meteorológicos durante las tres campañas de cultivos realizados (Tmax: temperatura máxima, Tmin: temperatura mínima) 


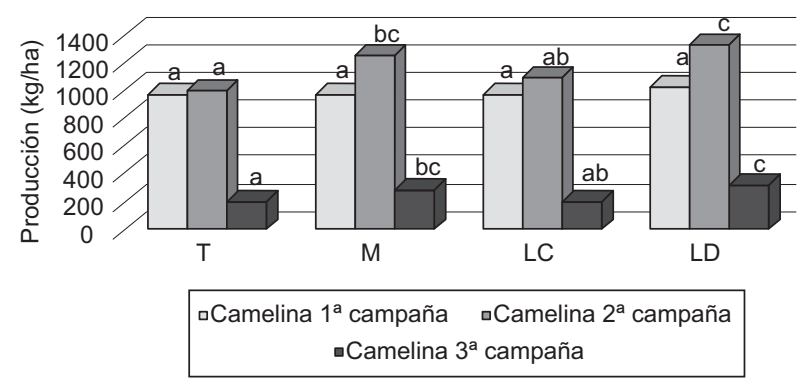

Fig. 2. Rendimientos en granos de la camelina según el tratamiento (T: testigo, M: fertilización mineral, LC: lodo compostado, LD: lodo deshidratado)

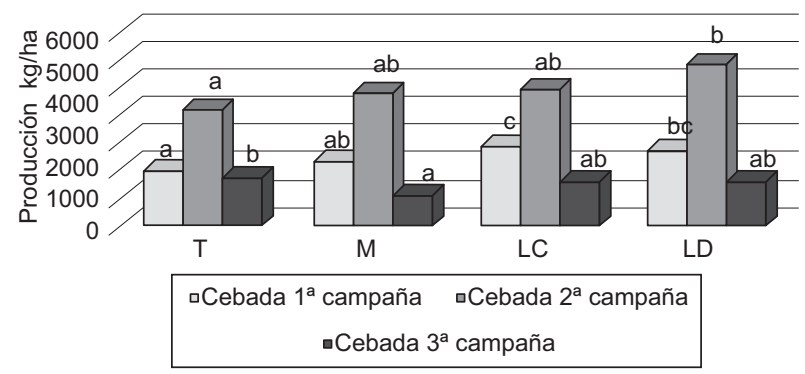

Fig. 3. Rendimientos en grano de la cebada según el tratamiento (T: testigo, M: fertilización mineral, LC: lodo compostado, LD: lodo deshidratado)

de a biomasa aérea para la camelina en cada uno de los tratamientos, así como el porcentaje de proteína y grasa en el grano para cada campaña (Cuadro IV).
En la primera campaña el mayor rendimiento en biomasa fue para el LC con diferencias significativas frente al resto; en cambio, para el rendimiento en grano y peso de mil granos no se encontraron diferencias. En cuanto al porcentaje de grasa bruta, los mayores valores fueron para $\mathrm{T}$ y LC frente a M y LD; lo contrario ocurrió para el porcentaje de proteína bruta, datos que concuerdan con los de otros autores como Solís et al. (2013) y Malhi et al. (2014), quienes reseñaron que al aumentar la fertilización nitrogenada aumenta la proteína y disminuye la grasa, lo que ocurrió tanto en la segunda como en la tercera campaña. En la segunda campaña el mayor rendimiento en biomasa fue para LC y en grano para LD y M. En la tercera campaña los mayores rendimientos en biomasa fueron para LD y $\mathrm{M}$ frente al resto, y el menor para el T. El rendimiento en grano tuvo un comportamiento muy similar a la biomasa, no así en el peso de mil granos, donde el mayor valor fue para el T frente al resto. El LD fue el tratamiento que presentó los mayores rendimientos en grano en las tres campañas. Se estudiaron los mismos parámetros para la cebada (Cuadro V).

En la primera campaña, los menores rendimientos en biomasa y grano fueron para el $\mathrm{T}$ frente a LD, sin deferencias en el porcentaje de proteína bruta, en tanto que el menor peso de mil granos fue para el $\mathrm{T}$ frente al resto. En la segunda campaña el T mostró igualmente el menor rendimiento en biomasa frente al resto y en grano frente al $\mathrm{LD}$; el porcentaje de

CUADRO IV. PROMEDIOS DE RENDIMIENTO EN BIOMASA AÉREA DE LA CAMELINA, RENDIMIENTO DE GRANO, PESO DE 1000 GRANOS Y CONTENIDO DE GRASA Y PROTEÍNA, EN LOS DIFERENTES TRATAMIENTOS POR CAMPAÑA

\begin{tabular}{|c|c|c|c|c|c|c|}
\hline Campaña & Tratamientos & $\begin{array}{c}\text { Rendimiento en } \\
\text { biomasa aérea } \mathrm{kg} / \mathrm{ha}\end{array}$ & $\begin{array}{l}\text { Rendimiento de } \\
\text { grano } \mathrm{kg} / \mathrm{ha}\end{array}$ & $\begin{array}{c}\text { Grasa } \\
\text { bruta } \%\end{array}$ & $\begin{array}{l}\text { Proteína } \\
\text { bruta \% }\end{array}$ & $\begin{array}{l}\text { Peso de mil } \\
\text { granos }(\mathrm{g})\end{array}$ \\
\hline \multirow{4}{*}{1} & $\mathrm{~T}$ & $1906.69 \mathrm{a}$ & $990.02 \mathrm{a}$ & $35.02 \mathrm{~b}$ & $27.31 \mathrm{a}$ & $1.06 \mathrm{a}$ \\
\hline & $\mathrm{M}$ & $1856.93 \mathrm{a}$ & $928.35 \mathrm{a}$ & $34.06 \mathrm{a}$ & $28.06 \mathrm{~b}$ & $1.04 \mathrm{a}$ \\
\hline & $\mathrm{LC}$ & $2655.74 \mathrm{~b}$ & $953.35 \mathrm{a}$ & $34.79 \mathrm{~b}$ & $27.33 \mathrm{a}$ & $1.04 \mathrm{a}$ \\
\hline & LD & $1822.41 \mathrm{a}$ & $1036.69 \mathrm{a}$ & $34.01 \mathrm{a}$ & $28.41 \mathrm{~b}$ & $1.05 \mathrm{a}$ \\
\hline \multirow{4}{*}{2} & $\mathrm{~T}$ & $5357.14 \mathrm{a}$ & $1012.84 \mathrm{a}$ & $38.15 \mathrm{~b}$ & $24.67 \mathrm{a}$ & $1.21 \mathrm{a}$ \\
\hline & $\mathrm{M}$ & $5833.33 \mathrm{a}$ & $1261.36 \mathrm{~b}$ & $35.51 \mathrm{a}$ & $26.21 \mathrm{~b}$ & $1.11 \mathrm{a}$ \\
\hline & $\mathrm{LC}$ & $6250.01 \mathrm{a}$ & $1110.17 \mathrm{a}$ & $38.05 \mathrm{~b}$ & $24.59 \mathrm{a}$ & $1.21 \mathrm{a}$ \\
\hline & LD & $9345.24 b$ & $1343.63 \mathrm{~b}$ & $34.91 \mathrm{a}$ & $26.86 \mathrm{~b}$ & $1.08 \mathrm{a}$ \\
\hline \multirow{4}{*}{3} & $\mathrm{~T}$ & $1005.95 \mathrm{a}$ & 203.77 a & $34.11 \mathrm{~b}$ & $26.08 \mathrm{a}$ & $0.87 \mathrm{~b}$ \\
\hline & $\mathrm{M}$ & $2322.68 \mathrm{c}$ & $278.08 \mathrm{bc}$ & $32.54 \mathrm{a}$ & $27.53 b$ & $0.78 \mathrm{a}$ \\
\hline & $\mathrm{LC}$ & $1666.67 \mathrm{~b}$ & $248.44 \mathrm{~b}$ & $33.93 \mathrm{~b}$ & $26.15 \mathrm{a}$ & $0.77 \mathrm{a}$ \\
\hline & LD & $2541.65 \mathrm{c}$ & $306.94 \mathrm{c}$ & $32.92 \mathrm{a}$ & $27.05 \mathrm{~b}$ & $0.77 \mathrm{a}$ \\
\hline
\end{tabular}

T: testigo, M: fertilización mineral, LC: lodo compostado, LD: lodo deshidratado

Nota: Los valores con letras iguales en cada columna y campaña no presentan diferencias significativas según la prueba de Duncan $(\alpha=0.05)$ 
CUADRO V. PROMEDIOS DE RENDIMIENTO EN BIOMASAAÉREA DE LA CEBADA, RENDIMIENTO DE GRANO, PESO DE 1000 GRANOS Y CONTENIDO DE GRASA Y PROTEÍNA, EN LOS DIFERENTES TRATAMIENTOS POR CAMPAÑA

\begin{tabular}{|c|c|c|c|c|c|}
\hline Campaña & Tratamientos & $\begin{array}{c}\text { Rendimiento en } \\
\text { biomasa aérea } \mathrm{kg} / \mathrm{ha}\end{array}$ & $\begin{array}{l}\text { Rendimiento } \\
\text { de grano } \mathrm{kg} / \mathrm{ha}\end{array}$ & $\begin{array}{l}\text { Proteína } \\
\text { bruta \% }\end{array}$ & $\begin{array}{l}\text { Peso de mil } \\
\text { granos }(\mathrm{g})\end{array}$ \\
\hline \multirow{4}{*}{1} & $\mathrm{~T}$ & $7543.21 \mathrm{a}$ & $1719.51 \mathrm{a}$ & $15.07 \mathrm{a}$ & $20.02 \mathrm{a}$ \\
\hline & $\mathrm{M}$ & $7853.09 \mathrm{ab}$ & $2035.01 \mathrm{ab}$ & $14.17 \mathrm{a}$ & $24.96 \mathrm{~b}$ \\
\hline & $\mathrm{LC}$ & $7409.88 \mathrm{a}$ & $2492.67 \mathrm{~b}$ & $15.45 \mathrm{a}$ & $28.01 \mathrm{c}$ \\
\hline & LD & $8530.86 \mathrm{~b}$ & $2374.68 \mathrm{~b}$ & $15.05 \mathrm{a}$ & $26.02 \mathrm{~b}$ \\
\hline \multirow{4}{*}{2} & $\mathrm{~T}$ & $10119.01 \mathrm{a}$ & $3725.71 \mathrm{a}$ & $9.41 \mathrm{a}$ & $34.51 \mathrm{c}$ \\
\hline & $\mathrm{M}$ & $15238.11 \mathrm{~b}$ & $4200.27 \mathrm{ab}$ & $11.99 \mathrm{~b}$ & $29.16 \mathrm{a}$ \\
\hline & $\mathrm{LC}$ & $13690.51 \mathrm{~b}$ & $4301.88 \mathrm{ab}$ & $9.24 \mathrm{a}$ & $32.24 \mathrm{~b}$ \\
\hline & LD & $15447.61 \mathrm{~b}$ & $5116.49 \mathrm{~b}$ & $10.75 \mathrm{ab}$ & $28.55 \mathrm{a}$ \\
\hline \multirow{4}{*}{3} & $\mathrm{~T}$ & 8720.24 bc & $1536.52 \mathrm{~b}$ & $16.41 \mathrm{a}$ & $15.83 \mathrm{~b}$ \\
\hline & M & $6541.67 \mathrm{a}$ & $892.95 \mathrm{a}$ & $18.64 \mathrm{~b}$ & $10.01 \mathrm{a}$ \\
\hline & $\mathrm{LC}$ & $7642.86 \mathrm{ab}$ & $1349.88 \mathrm{~b}$ & $16.03 \mathrm{a}$ & $18.92 \mathrm{~b}$ \\
\hline & LD & $9833.33 \mathrm{c}$ & $1323.69 \mathrm{~b}$ & $18.76 \mathrm{~b}$ & $14.52 \mathrm{ab}$ \\
\hline
\end{tabular}

T: testigo, M: fertilización mineral, LC: lodo compostado, LD: lodo deshidratado Nota: Los valores con letras iguales en cada columna y campaña no presentan diferencias significativas según la prueba de Duncan $(\alpha=0.05)$

proteína bruta mayor fue para M y el mayor peso de mil granos para el T. En la tercera campaña el mayor rendimiento en biomasa fue para el LD frente a la $\mathrm{M}$ y en grano la $\mathrm{M}$ fue menor al resto; en cambio, la M junto al LD mostraron los mayores valores en el porcentaje de proteína bruta frente al resto y en el peso de mil granos, su comportamiento fue opuesto con los mayores valores para el LC y T. Como aprovechamiento de la biomasa o restos de cultivo (paja) el tratamiento con mayor rendimiento en todas las campañas fue el LD. El rendimiento en grano del cultivo se ve favorecido por los tratamientos fertilizantes frente al $\mathrm{T}$ excepto en la tercera campaña donde éste presentó el mayor rendimiento junto con LC y LD frente a la M, influenciado por las condiciones climatológicas ya mencionadas.

En la bibliografía consultada aparecen índices y parámetros para el estudio de la cebada, como la relación entre producción biológica y agronómica, que se define como índice de cosecha (Mengel y Kirkby 2001, Hernández 2006). Éste representa la fracción de peso seco total de la planta que se encuentra en forma de grano y refleja la eficacia del cultivo en el uso del fertilizante, ya que un índice de cosecha elevado indica una distribución mejor de compuestos asimilados dentro de la planta, siendo mayor en el grano que en la paja (Molina 1989).

La producción de grano en los cultivos puede estar afectada por varios parámetros, lo que permite estudiar la contribución de las distintas partes de la planta a la producción de grano y la causa de variación en la cosecha final (Molina 1989, Hernández 2006). El rendimiento de grano puede expresarse como el producto entre el número de espigas por unidad de superficie, el número de granos por espiga y el peso medio del grano. El producto de los dos primeros se denomina factor de acumulación o capacidad de almacenamiento y equivale al número de granos por unidad de superficie, mientras que el peso medio por grano indica la medida en que esta capacidad de almacenamiento se transforma en rendimiento final.

Según los datos obtenidos (cuadro VI), la primera campaña mostró que el comportamiento de la fertilización estuvo en todos los parámetros por encima del $\mathrm{T}$ excepto en el factor de acumulación. En cambio, en la segunda campaña, en algunos parámetros como IC y peso específico del grano, el T no presento diferencias significativas con la fertilización orgánica, e incluso fue superior en el peso de mil granos. También se observaron diferencias significativas entre el LC y LD, correspondiendo a este último los valores mayores en número de espigas por $\mathrm{m}^{2} \mathrm{y}$ factor de acumulación a pesar de su única aplicación en el primer ciclo; lo contrario ocurrió respecto del peso de mil granos. En la tercera campaña los mayores índices de cosecha y peso de mil granos fueron para $\mathrm{T}$ y LC frente a M, y se encontraron diferencias significativas en el número de espigas por $\mathrm{m}^{2} \mathrm{y}$ factor de acumulación entre el $\mathrm{T}$ y LD frente al LC y la M. 
CUADRO VI. PROMEDIO DE LOS ÍNDICES DE COSECHA DE LA CEBADA, NÚMERO DE ESPIGAS, FACTOR DE ACUMULACIÓN Y PESO ESPECÍFICO DEL GRANO EN LOS DIFERENTES TRATAMIENTOS POR CAMPAÑA

\begin{tabular}{|c|c|c|c|c|c|}
\hline Campaña & Tratamientos & $\begin{array}{c}\text { Índice de } \\
\text { cosecha }\end{array}$ & $\begin{array}{l}\text { Número de espigas } \\
\left(\text { espigas } / \mathrm{m}^{2}\right)\end{array}$ & $\begin{array}{l}\text { Factor de acumulación } \\
\quad\left(\text { granos } / \mathrm{m}^{2}\right)\end{array}$ & $\begin{array}{l}\text { Peso específico del } \\
\text { grano }\left(\mathrm{kg} / \mathrm{m}^{3}\right)\end{array}$ \\
\hline \multirow{4}{*}{1} & $\mathrm{~T}$ & $0.14 \mathrm{a}$ & $630.66 \mathrm{a}$ & $8613.67 \mathrm{ab}$ & $464.71 \mathrm{a}$ \\
\hline & M & $0.26 \mathrm{c}$ & $683.01 \mathrm{~b}$ & $7869.93 \mathrm{a}$ & $541.07 \mathrm{ab}$ \\
\hline & $\mathrm{LC}$ & $0.24 \mathrm{bc}$ & $719.01 \mathrm{~b}$ & $8914.27 \mathrm{~b}$ & $607.14 \mathrm{~b}$ \\
\hline & LD & $0.20 \mathrm{~b}$ & $707.33 \mathrm{~b}$ & $8851.31 \mathrm{~b}$ & $539.63 \mathrm{ab}$ \\
\hline \multirow{4}{*}{2} & $\mathrm{~T}$ & $0.36 \mathrm{~b}$ & $639.28 \mathrm{a}$ & $10915.71 \mathrm{a}$ & $635.58 \mathrm{~b}$ \\
\hline & $\mathrm{M}$ & $0.28 \mathrm{a}$ & $1032.14 \mathrm{c}$ & $14801.03 \mathrm{~b}$ & $563.37 \mathrm{a}$ \\
\hline & $\mathrm{LC}$ & $0.33 \mathrm{ab}$ & $921.19 \mathrm{~b}$ & $13349.31 \mathrm{~b}$ & $670.41 \mathrm{~b}$ \\
\hline & LD & $0.36 \mathrm{~b}$ & $1034.52 \mathrm{c}$ & $17951.11 \mathrm{c}$ & $615.58 \mathrm{ab}$ \\
\hline \multirow{4}{*}{3} & $\mathrm{~T}$ & $0.18 \mathrm{~b}$ & $584.52 \mathrm{~b}$ & $9589.29 \mathrm{~b}$ & $481.44 \mathrm{a}$ \\
\hline & $\mathrm{M}$ & $0.11 \mathrm{a}$ & $487.62 \mathrm{a}$ & $7434.58 \mathrm{a}$ & $491.79 \mathrm{a}$ \\
\hline & $\mathrm{LC}$ & $0.17 \mathrm{~b}$ & $496.43 \mathrm{a}$ & $7260.36 \mathrm{a}$ & $468.41 \mathrm{a}$ \\
\hline & LD & $0.14 \mathrm{ab}$ & $598.33 \mathrm{~b}$ & $9291.19 \mathrm{~b}$ & $490.91 \mathrm{a}$ \\
\hline
\end{tabular}

T: testigo, M: fertilización mineral, LC: lodo compostado, LD: lodo deshidratado Nota: Los valores con letras iguales en cada columna y campaña no presentan diferencias significativas según la prueba de Duncan $(\alpha=0.05)$

En cuanto a las diferencias entre campañas, los menores valores para ambos cultivos en el caso de producción (figuras 2 y 3 ) y peso de mil granos, fueron para la tercera campaña, seguida de la primera y segunda, con diferencias significativas $(\mathrm{p} \leq 0.05)$ entre ellas. No se encontraron diferencias significativas para la interacción tratamiento*campaña. Respecto de la biomasa, la camelina no presentó diferencias entre la primera y tercera campaña, siendo éstas significativamente menores que la segunda. Con relación a la cebada hubo diferencias significativas entre todas las campañas; la menor fue la primera, seguida de la tercera y la segunda (esta última con la mayor producción). En cuanto a la camelina, el menor valor para la proteína bruta se obtuvo en la segunda campaña, con diferencias significativas frente al resto de las compañas y un resultado contrario para el porcentaje de grasa bruta. Para el porcentaje de proteína bruta en la cebada también se encontraron diferencias significativas entre campañas, siendo de mayor a menor la tercera, la primera y por último la segunda.

La evaluación de la calidad del grano obtenido en cada cosecha se realizó en función del peso del grano (peso de mil granos). Según Molina (1989), el peso del grano está en función de la etapa de maduración y de la velocidad de crecimiento del grano, y se considera un componente de la cosecha relativamente estable en condiciones normales de cultivo. Un peso de grano menor indica un descenso en los aportes de compuestos asimilados, debido especialmente a condiciones ambientales desfavorables como mayor sequía y temperatura al final del ciclo del cultivo (Hernández 2006). Con relación a la cebada se observó que la sequía durante el periodo de llenado del grano fue también responsable de las respuestas genotípicas diferenciales encontradas en el peso unitario del grano (Voltas et al. 1999). Se pudo constatar que en los tratamientos de la tercera campaña con cantidades de nutrientes mayores (como $\mathrm{M}$, cuyo rendimiento de mil granos [10.1 g] fue significativamente menor $[\mathrm{p} \leq 0.05]$ a $\mathrm{T}[15.83 \mathrm{~g}])$, las condiciones ambientales tuvieron efectos similares en el desarrollo del cultivo a los reportados por estos autores.

\section{Efectos sobre el suelo}

Se realizaron dos tipos de estudios a profundidades de 0-30 y 30-60 cm: uno para observar diferencias en el suelo tras el cultivo de la camelina frente al de cebada, y otro para observar las diferencias tras las tres campañas con las diferentes rotaciones de ambos cultivos.

Según el cultivo desarrollado, se encontraron diferencias significativas en el suelo $(\mathrm{p} \leq 0.05)$ en los primeros $30 \mathrm{~cm}$ para conductividad eléctrica, la cual fue menor tras el cultivo de camelina $(0.13 \mathrm{dS} / \mathrm{m})$ frente al de cebada $(0.18 \mathrm{dS} / \mathrm{m})$ en la M. Los valores de nitrógeno Kjeldahl fueron mayores después del cultivo de camelina en $\mathrm{T}$ y $\mathrm{LD}$, con valores de $0.1 \mathrm{y}$ $0.14 \%$ frente a 0.07 y $0.11 \%$, respectivamente, tras 
el cultivo de cebada. El fósforo asimilable presentó mayores cantidades tras el cultivo de camelina con $82.2 \mathrm{mg} / \mathrm{kg}$ frente a $46.6 \mathrm{mg} / \mathrm{kg}$ tras el de cebada en el tratamiento LD. Para el potasio asimilable, tras el cultivo de camelina el suelo presentó valores de 213, 258,227 y $232 \mathrm{mg} / \mathrm{kg}$ para T, M, LC y LD, respectivamente, que fueron significativamente menores en todos los tratamientos, frente al suelo tras el cultivo de cebada, que tuvo valores de 338, 423, 360 y 334 $\mathrm{mg} / \mathrm{kg}$ para T, M, LC y LD, respectivamente. El potasio total fue menor únicamente en el testigo, con un valor de $0.48 \%$ frente a $0.62 \%$ tras el cultivo de cebada. En cuanto al resto de los elementos, sólo el Ca presentó valores significativamente mayores tras el cultivo de camelina con 4.43 y $3.77 \%$ en los tratamientos LC y LD, respectivamente, frente a 2.4 y $2.28 \%$ tras el cultivo de cebada.

En el estrato de 30 a $60 \mathrm{~cm}$, tras el cultivo de cebada, la $\mathrm{CE}$ y el nitrato fueron menores en el tratamiento $\mathrm{LC}$, con valores de $0.125 \mathrm{dS} / \mathrm{m}$ y $15,41 \mathrm{mg} / \mathrm{kg}$, frente a $0.177 \mathrm{dS} / \mathrm{m}$ y $35.33 \mathrm{mg} / \mathrm{kg}$ tras el cultivo de camelina. El potasio asimilable fue mayor tras el cultivo de cebada en T con $286.83 \mathrm{mg} / \mathrm{kg}$ frente a $193.66 \mathrm{mg} /$ $\mathrm{kg}$ tras el cultivo de camelina, y el calcio fue mayor tras el cultivo de camelina en los tratamientos LC y $\mathrm{LD}$, con valores de 5.17 y $4.99 \%$, respectivamente, frente a 2.74 y $2.72 \%$ tras el cultivo de cebada.

Respecto al estudio para conocer la evolución del suelo tras las tres campañas (Cuadro II) por tratamientos, se encontraron diferencias significativas $(\mathrm{p} \leq 0.05)$ en los parámetros estudiados según avanzó el ensayo para los primeros $30 \mathrm{~cm}$ de profundidad.

La conductividad eléctrica disminuyó tras las campañas en los tratamientos T, LC y LD, mientras que para la M se observó un aumento de $0.04 \mathrm{dS} / \mathrm{m}$; sin embargo, no presentó diferencias significativas entre campañas.

La materia orgánica mostró incremento en los tratamientos LC y LD, datos concordantes con los de autores como Beltrán et al. (2005) y Fernández et al. (2009). En cuanto a los macronutrientes, se observó una disminución del nitrógeno para el testigo y un aumento para M y LD. Con relación a las formas del nitrógeno, se observaron valores significativamente mayores para nitrógeno amoniacal $\left(\mathrm{N}_{-} \mathrm{NH}_{4}{ }^{+}\right)$al final de la primera campaña para todos los tratamientos; con relación al nitrógeno proveniente de nitratos $\left(\mathrm{N}^{-\mathrm{NO}_{3}}{ }^{-}\right)$ se observaron disminuciones en T, LC y LD, mientras que en la $\mathrm{M}$ no se registraron cambios excepto en la última campaña, cuando aumentó (posiblemente por el mal año climatológico, ya que los cultivos fueron incapaces de tomar el $\mathrm{N}_{-} \mathrm{NO}_{3}{ }^{-}$del suelo). El fósforo asimilable mostró un aumento tras las campañas en todos los tratamientos, excepto en $\mathrm{T}$, con valores iniciales de $55,51,52$ y $57 \mathrm{mg} / \mathrm{kg}$ frente a los valores finales de $56,92,92$, y $75 \mathrm{mg} / \mathrm{kg}$ para T, M, LC y LD, respectivamente, efecto observado también por Miralles et al. (2003). En cambio, para el fósforo total, el mayor aumento se dio en el tratamiento $\mathrm{M}$. El potasio asimilable presentó un ligero aumento no significativo, aunque el potasio total sí presentó diferencias significativas, las cuales fueron mayores en las dos últimas campañas para todos los tratamientos.

Al igual que el $\mathrm{N}_{-} \mathrm{NH}_{4}{ }^{+}$, el calcio y magnesio, fueron significativamente mayores al final de la primera campaña; después no presentaron diferencias respecto a los valores iniciales. El sodio aumentó con el paso de las campañas en todos los tratamientos.

En cuanto a los metales pesados, se produjeron pequeñas variaciones en todos los tratamientos incluido $\mathrm{T}$, por lo que dichas diferencias no se debieron exclusivamente a los tratamientos aplicados. Ninguna de estas variaciones presentó diferencias significativas entre el inicio y al final de las tres campañas, encontrándose siempre dentro de los niveles permitidos por la legislación vigente en el Real Decreto 1310/1990 (BOE 1990).

Al estudiar el suelo a una profundidad de 30 a $60 \mathrm{~cm}$ para comprobar posibles movimientos de macronutrientes en la vertical, se encontraron diferencias significativas $(p \leq 0.05)$ en varios de los parámetros estudiados. La conductividad eléctrica en T y LD mostró una disminución entre el inicio de los tratamientos, con valores de 0.131 y $0.152 \mathrm{dS} / \mathrm{m}$, y el final de las tres campañas, con valores de 0.111 y $0.114 \mathrm{dS} / \mathrm{m}$, respectivamente. En cuanto al nitrógeno, sólo se encontraron diferencias significativas para la forma $\mathrm{N}-\mathrm{NH}_{4}{ }^{+}$, que fue superior al final de las campañas en todos los tratamientos. En cambio, el $\mathrm{N}-\mathrm{NO}_{3}{ }^{-}$fue menor al final de las tres campañas, con valores de 12.11 y $10.61 \mathrm{mg} / \mathrm{kg}$ en los tratamientos T y LD, frente al inicio, con valores de 38.11 y 54.23 $\mathrm{mg} / \mathrm{kg}$, respectivamente, y mayor en el tratamiento $\mathrm{M}$, con un valor inicial de $36.23 \mathrm{mg} / \mathrm{kg}$ frente a 70.18 $\mathrm{mg} / \mathrm{kg}$ al final de las tres campañas. El potasio total presentó aumentos significativos en todos los tratamientos, con valores iniciales de $0.54,0.47,0.52$, y $0.45 \%$ para T, M, LC y LD, respectivamente, frente a valores finales de $0.61,0.64,0.59$ y $0.59 \%$.

Respecto a los metales pesados a esta profundidad del suelo, igual que en los primeros $30 \mathrm{~cm}$, no se encontraron diferencias significativas tras las tres campañas.

De acuerdo con los resultados obtenidos, se puede decir que la aplicación al suelo de lodos de depuradora puede tener efectos beneficiosos para la 
agricultura (Tarazona et al. 1996), pero su uso debe ser controlado y cumplir en todo momento con la legislación vigente y los códigos agronómicos y de buenas prácticas agrarias.

Si se tienen en cuenta los costes de fertilización entre tratamientos, se observó que los tratamientos T y LD presentaron los mayores beneficios después de las tres campañas con 994 y $950 € /$ ha, respectivamente, seguidos del LC con $530 € /$ ha y por último el mineral con $383 € /$ ha para las tres campañas ensayadas.

\section{CONCLUSIONES}

Con base en los resultados de producción y la evolución de los compuestos estudiados en el suelo, se puede apreciar que la fertilización orgánica fue adecuada. En el caso de la fertilización mineral para el cultivo de camelina, deben realizarse más estudios para lograr un ajuste adecuado en las condiciones agroclimáticas registradas, ya que el mayor coste en la producción de esta planta es la fertilización nitrogenada; por tanto, la mayor prioridad es optimizarla para evitar la contaminación y la pérdida de nutrientes, así como lograr la reducción de costes y mayor rentabilidad.

\section{AGRADECIMIENTOS}

Este estudio se realizó gracias al contrato firmado entre el Instituto Nacional de Investigación y Tecnología Agraria y Alimentaria (INIA) y Camelina Company España. Los autores agradecen a Luis Martínez Cortizo, Rosario Miralles de Imperial Hornedo y Jesús García su inestimable colaboración en la realización de este estudio.

\section{REFERENCIAS}

Arlabosse P., Ferrasse J.H., Lecomte D., Crine M., Dumont Y. y Léonard A. (2012). Efficient sludge thermal processing: from drying to thermal valorization. En: Modern drying technology, energy savings, vol. 4 (Tsotsas E. y Mujumdar A.S., Eds.). WileyVCH, Weinheim, Alemania, 295-329. https://doi. org/10.1002/9783527631681.ch8

Beltrán E.M., Miralles de Imperial R., Porcel M.A., Martin J.V., Beringola M.L., Calvo R. y Delgado M.M. (2005). Influencia de la fertilización con lodos de depuradora compostados en las propiedades químicas del suelo de dos olivares. Rev. Int. Contam. Ambie. 21 (3), 143-150.
Bennamoun L., Arlabosse P. y Léonard A. (2013). Review on fundamental aspect of application of drying process to wastewater sludge. Renew. Sust. Energ. Rev. 28, 29-43. https://doi.org/10.1016/j.rser.2013.07.043

BOE (1990). Real Decreto 1310/1990. Regula la utilización de los lodos de depuración en el sector agrario. Ministerio de Agricultura, Pesca y Alimentación. Boletín Oficial del Estado, 1 de noviembre.

BOE (1995). Real Decreto-Ley 11/1995. Se establecen las normas aplicables al tratamiento de las aguas residuales urbanas. Jefatura del Estado. Boletín Oficial del Estado, 30 de diciembre.

BOE (2013). Orden AAA/1072/2013. Utilización de lodos de depuración en el sector agrario. Ministerio de Agricultura, Alimentación y Medio Ambiente. Boletín Oficial del Estado, 14 de junio.

Bobrecka-Jamro M.C. (2017). The effects of varied plant density and nitrogen fertilization on quantity and quality yield of camelina sativa L. Emir. J. Food Agr. 29 (12), 988-993. https://doi.org/10.9755/ejfa.2017. v29.i12.1569

CCE (1991). Directiva 91/271/CEE. Sobre el tratamiento de las aguas residuales urbanas. Consejo de las Comunidades Europeas. Diario Oficial de las Comunidades Europeas, 30 de mayo.

Dentel S.K. (2004). Contaminants in sludge: Implications for management policies and land application. Water Sci. Technol. 49 (10), 21-29. https://doi.org/10.2166/ wst.2004.0600

Fytili D. y Zabaniotou A. (2008). Utilization of sewage sludge in EU application of old and new methods. A review. Renew. Sust. Energ. Rev. 12 (1), 116-140. https://doi.org/10.1016/j.rser.2006.05.014

Fernández J.M., Plaza C., García-Gil J.C. y Polo A. (2009). Biochemical properties and barley yield in a semiarid Mediterranean soil amended with two kinds of sewage sludge. Appl. Soil Ecol. 42 (1), 18-24. https://doi. org/10.1016/j.apsoil.2009.01.006

Gesch R.W. (2014). Influence of genotype and sowing date on camelina growth and yield in the north central U.S. Ind. Crop. Prod. 54, 209-215. https://doi.org/10.1016/j. indcrop.2014.01.034

Hernández D. (2006). Utilización del purín de cerdo como enmienda orgánica en suelos degradados: valorización agronómica y ambiental. Tesis de Doctorado. Facultad de Ciencias, Universidad Autónoma de Madrid, Madrid, España, 257 pp.

Jiang Y. y Caldwell C.D. (2016). Effect of nitrogen fertilization on camelina seed yield, yield components, and downy mildew infection. Can. J. Plant Sci. 96 (1), 17-26. https://doi.org/10.1139/cjps-2014-0348

Kelessidis A. y Stasinakis A.S. (2012). Comparative study of the methods for the treatment and final disposal of 
sewage sludge in European countries. Waste Manage. 32 (6), 1186-1195. https://doi.org/10.1016/j.wasman.2012.01.012

Keshavarz-Afshar R., Mohammed Y.A. y Chen C. (2016). Enhanced efficiency nitrogen fertilizer effect on camelina production under conventional and conservation tillage practices. Ind. Crop. Prod. 94, 783-789. https:// doi.org/10.1016/j.indcrop.2016.09.043

Leonard A. (2011). Management of wastewater sludge's: A hot topic at the European level. J. Residuals Sci. Tech 8, 38-38.

Malhi S.S., Johnson E.N., Hall L.M., May W.E., Phelps S. y Nybo B. (2014). Effect of nitrogen fertilizer application on seed yield, N uptake, and seed quality of Camelina sativa. Can. J. Soil Sci. 94 (1), 35-47. https:// doi.org/10.4141/cjss2012-086

Mengel K. y Kirkby E. (2001). Principles of plant nutrition. 5a ed. Kluwer Academic publishers, Dordrecht, Países Bajos, 256 pp.

Miralles de Imperial R., Beltrán E.M., Porcel M.A., Beringola M.L., Martin J.V., Calvo R. y Delgado M.M. (2003). Disponibilidad de nutrientes por el aporte de tres tipos de lodo de estaciones depuradoras. Rev. Int. Contam. Ambie. 19 (3), 127-136.

Molina J.L. (1989). La cebada. Morfología, fisiología, genética, agronomía y usos industriales. Ministerio de Agricultura, Pesca y Alimentación. Madrid, España, $252 \mathrm{pp}$.

Pérez-Elvira S.I., Nieto P. y Fernández-Polanco F. (2006). Sludge minimisation technologies. Rev. Environ. Sci. Bio. 5 (4), 375-398. https://doi.org/10.1007/s11157005-5728-9

Pervez-Zamurrad J., Ghulam S. y Nazakat-Ullah K. (2010). Impact of climate change on wheat production: A case study of Pakistan. The Pakistan Development Review 49 (4), 799-822. https://doi.org/10.30541/ v49i4IIpp.799-822

Singh R.P. y Agrawal M. (2008). Potential benefits and risks of land application of sewage sludge. Waste Manage. 28 (2), 347-358. https://doi.org/10.1016/j. wasman.2006.12.010
Soil Survey Staff (2010). Keys to soil taxonomy. 7a ed. United States Department of Agriculture, Natural Resources Conservation Service. Washington, DC, EUA, 345 pp.

Solís A., Vidal I., Paulino L., Johnson B.L. y Berti M.T. (2013). Camelina seed yield response to nitrogen, sulphur, and phosphorus fertilizer in south Central Chile. Ind. Crop Prod. 44, 132-138. https://doi.org/10.1016/j. indcrop.2012.11.005

Tarazona F., Estela M., Pomares F. y Canet R. (1996). Efecto de los lodos de depuradora en la producción de hortalizas y las propiedades químicas del suelo. Investigación Agraria: Producción y Protección Vegetal 11 (1), 83-100.

Uggetti E., Ferrer I., Nielsen S., Arias C., Brix H. y García J. (2012). Characteristics of biosolids from sludge treatment wetlands for agricultural reuse. Ecol. Eng. 40, 210-216. https://doi.org/10.1016/j. ecoleng.2011.12.030

Voltas J., van Eeuwijk F.A., Sombrero A., Lafarga A., Igartua E. y Romagosa I. (1999). Integrating statistical and ecophysiological analyses of genotype by environment interaction for grain filling of barley I. Individual grain weight. Field Crop. Res. 62 (1), 63-74. https:// doi.org/10.1016/S0378-4290(99)00006-4

Warrick R. A. (1988). Carbon dioxide, climate change and agriculture. Geograp. J. 154 (2), 221-233. https://doi. org $/ 10.2307 / 633848$

Wojtkowiak R., Frackowiak P., Glazar K. y Zembrowski K. (2009). Production costs in a novel method of manufacture of the methyl esters from false flax (Camelina sativa $\mathrm{L}$.) oil for feed the piston compression-ignition engines. Journal of Research and Application in Agriculture Engineering 54 (4), 164-170.

Zubr J. (2007). Dietary fatty acids and amino acids of camelina sativa seed. J. Food Quality 26, 451-462. https://doi.org/10.1111/j.1745-4557.2003.tb00260.x 